\title{
Further evidence for population specific differences in the effect of DNA markers and gender on eye colour prediction in forensics
}

\author{
Ewelina Pośpiech ${ }^{1,2}$ • Joanna Karlowska-Pik ${ }^{3}$ Bartosz Ziemkiewicz ${ }^{3}$. \\ Magdalena Kukla $^{4}$ - Malgorzata Skowron ${ }^{5}$ - Anna Wojas-Pelc ${ }^{5}$ - Wojciech Branicki ${ }^{2}$
}

Received: 9 March 2016 / Accepted: 9 May 2016/Published online: 24 May 2016

(C) The Author(s) 2016. This article is published with open access at Springerlink.com

\begin{abstract}
The genetics of eye colour has been extensively studied over the past few years, and the identified polymorphisms have been applied with marked success in the field of Forensic DNA Phenotyping. A picture that arises from evaluation of the currently available eye colour prediction markers shows that only the analysis of HERC2-OCA2 complex has similar effectiveness in different populations, while the predictive potential of other loci may vary significantly. Moreover, the role of gender in the explanation of human eye colour variation should not be neglected in some populations. In the present study, we re-investigated the data for 1020 Polish individuals and using neural networks and logistic regression methods explored predictive capacity of IrisPlex SNPs and gender in this population sample. In general, neural networks provided higher prediction accuracy comparing to logistic regression (AUC increase by $0.02-0.06$ ). Four out of six IrisPlex SNPs were associated with eye colour in the studied population. HERC2 rs12913832, OCA2 rs 1800407 and
\end{abstract}

Ewelina Pośpiech, Joanna Karłowska-Pik and Bartosz Ziemkiewicz contributed equally to this work.

Ewelina Pośpiech

ewelina.pospiech@uj.edu.pl

1 Institute of Zoology, Faculty of Biology and Earth Sciences, Jagiellonian University, Kraków, Poland

2 Malopolska Centre of Biotechnology, Jagiellonian University, Kraków, Poland

3 Faculty of Mathematics and Computer Science, Nicolaus Copernicus University, Torun, Poland

4 Faculty of Biochemistry, Biophysics and Biotechnology, Jagiellonian University, Kraków, Poland

5 Department of Dermatology, Collegium Medicum of the Jagiellonian University, Kraków, Poland
SLC24A4 rs12896399 were found to be the most important eye colour predictors $(p<0.007)$ while the effect of rs16891982 in SLC45A2 was less significant. Gender was found to be significantly associated with eye colour with males having $\sim 1.5$ higher odds for blue eye colour comparing to females $(p=0.002)$ and was ranked as the third most important factor in blue/non-blue eye colour determination. However, the implementation of gender into the developed prediction models had marginal and ambiguous impact on the overall accuracy of prediction confirming that the effect of gender on eye colour in this population is small. Our study indicated the advantage of neural networks in prediction modeling in forensics and provided additional evidence for population specific differences in the predictive importance of the IrisPlex SNPs and gender.

Keywords Forensic DNA Phenotyping (FDP) · Eye colour prediction · Gender effect · IrisPlex SNPs · Neural networks · Logistic regression

\section{Introduction}

Genetic prediction of human traits known as Forensic DNA Phenotyping (FDP) has enabled further development of methods and tools offered for intelligence purposes. Detailed description of appearance of an unknown individual based on DNA analysis of biological material can streamline the process of investigation in criminal cases without any suspects or entries in DNA profile databases [1-3]. Eye colour has been one of the first traits of human appearance successfully applied in the field of FDP. Among several available eye-colour prediction tools, the IrisPlex model is based on the largest number of samples ( $>9000$ Europeans) [4] and was successfully validated by a multicenter EDNAP study [5]. IrisPlex 
involves examination of six DNA variants, namely rs12913832 in HERC2, rs1800407 in OCA2, rs12896399 in SLC24A4, rs16891982 in SLC45A2, rs1393350 in TYR and rs12203592 in IRF4 and has been introduced to predict blue and brown eye colour precisely [6]. There is significant evidence from functional studies that the position rs 12913832 in HERC2 plays a crucial role in blue eye colour determination [7], and this position has also been confirmed as the key eye colour predictor in various population samples. Predictive capacity of the remaining IrisPlex SNPs is not so obvious and shows significant variation depending on a study population [8-12]. Pietroni et al., for instance, argued that three SNPs: rs12913832, rs 1800407 and rs16891982 are the only informative markers among the six IrisPlex polymorphisms [13]. Position rs12203592 is regarded as the weakest predictor, and its association with eye colour has not been confirmed in several study samples $[8,10,12,14]$. In our population sample of 718 individuals from Poland, only four IrisPlex SNPs have been found to be associated with eye colour, with IRF4 and TYR unimportant for prediction [11].

Moreover, the examination of a Spanish population sample has unexpectedly detected the significance of gender in the explanation of human eye colour variation [9]. Gender has been found to explain discrepancies in eye colour prediction based on HERC2 rs12913832 polymorphism indicating that females tend to have darker eye colour than males when comparing the same genotypes. The intriguing observation based on the Spanish samples has been later confirmed in Italian population where gender has been ranked as the second most important predictor [13]. However, this effect has not been confirmed in samples from Denmark and Sweden [13]. Moreover, gender has been found not to improve the prediction of eye colour when incorporated into the original IrisPlex model [15] suggesting that the effect of gender on eye colour may be population specific and stronger in populations of southern Europe.

In the present study, the role of the six IrisPlex SNPs and gender for eye colour prediction was investigated in the population of 1020 previously genotyped individuals from Poland using neural networks (NN) and logistic regression modeling. Additionally, the IrisPlex SNPs were analysed with the available online IrisPlex calculator in order to compare its predictive performance in males and females.

\section{Materials and methods}

\section{Population samples and genotyping}

Samples involved 1020 unrelated individuals ( $>18$ years old) from Poland previously genotyped and interpreted using different statistical methods $[11,16]$. The study cohort included 420 males $(41.2 \%)$ and 597 females $(58.5 \%)$. No data about gender was available for the remaining three samples $(0.3 \%)$. Eye colour of the participants has been assessed by a physician specializing in dermatology and categorized as blue, green, hazel and brown. Written informed consent was obtained from all the samples donors, and the study protocol was approved by the Ethics Committee of the Jagiellonian University in Krakow (KBET/17/B/2005) and the Commission on Bioethics of the Regional Board of Medical Doctors in Krakow (48 KBL/OIL/2008). The genotyping procedure for the total number of 24 SNPs in 11 genes has been described in Pośpiech et al. [16].

\section{Evaluation of the effect of SNPs and gender on eye colour in the study population}

The genotypes for the six IrisPlex SNPs (rs12913832, rs1800407, rs 12896399 , rs 16891982 , rs 1393350 , rs12203592), gender and eye colours were retrieved and subjected to association testing which involved the pooled training and testing set from the previous study [11] (the total number of 1020 individuals) and prediction modeling using neural networks and logistic regression approaches. Analyses were performed with IBM SPSS Statistics v. 23 (SPSS Inc., Chicago, IL, USA). The bundle includes IBM SPSS Regression and IBM SPSS Neural Networks modules.

\section{Dependence testing}

The dependencies between eye colour (defined as blue/green/ hazel/brown) and gender were tested using Pearson's $\chi^{2}$ test. Pearson's contingency coefficient (more precisely its adjusted version with maximal value equal to 1 ) and Cramér's V coefficient were calculated to evaluate the strength of association of eye colour and all independent variables (6 IrisPlex SNPs and gender), all of them treated as categorical attributes [17].

\section{Power of the sample size}

Minimal values of odds ratios (ORs) detectable with a power of at least $80 \%$ were assessed with Fisher's exact test using power and sample size program (PS Program) v.3.1.2 (http:// biostat.mc.vanderbilt.edu/wiki/Main/PowerSampleSize).

\section{Association testing}

Binary logistic regression was applied to evaluate the association of particular variables with eye colour defined as blue/non-blue. Analyses were performed on the entire set of 1020 samples. Variables were tested as single factors (univariate logistic regression analysis) and then analysed simultaneously (multivariate logistic regression analysis) to assess the influence of gender on the six IrisPlex SNPs effects. Allelic ORs with $95 \%$ confidence intervals (CIs) and 
respective $p$ values were estimated for minor alleles categorized in an additive manner. The seven tested variables were ranked according to their importance using $-2 \log$ likelihood of reduced model statistic. The proportion of total variance in eye colour explained by the tested variables was estimated using Nagelkerke pseudo- $R^{2}$ statistic. Statistical significance was set at $p$ value lower than 0.05 . However, the results of association testing considering Bonferroni correction for multiple comparisons were also discussed $(p<0.007)$.

\section{Prediction modeling}

The entire set of 1020 individuals was used to develop prediction models and test the effect of particular SNPs and gender on prediction. A few prediction methods, including logistic regression, neural networks, classification and regression trees and random forests, were used to build models that were tested using tenfold cross-validation procedure. For this purpose, the data was split randomly into 10 equal-sized parts (identically for all tested models). For each $k(k=1,2, \ldots, 10)$ the $k$ th part was excluded, and the model was built using the data from the others $k-1$ parts. Then the prediction error was calculated on the excluded $k$ th part of the data. The final prediction error was estimated by the mean of errors of 10 models built in the cross-validation procedure. Because its lowest values were achieved for neural networks and well known logistic regression procedure, we decided to concentrate only on these two prediction methods.

By the neural network, we mean multilayer perceptron (MLP) with one hidden layer and an automatically selected number of neurons. The activation functions were hyperbolic tangent for the hidden layer and softmax for the output layer. Synaptic weights were updated after passing all training data (batch training type). As an optimization algorithm, we used scaled conjugate gradient with default initial parameters of IBM SPSS Statistics. The multinomial logistic regression model was developed using block entry of variables (entry value 0.05 ). The utility of the particular IrisPlex SNPs and gender for eye colour prediction in both cases was assessed by the sequential implementation of variables into the prediction models and the calculation of prediction accuracy parameters including area under the ROC curve (AUC), sensitivity and specificity [e.g. [18, 19]. All the described analyses were performed with IBM SPSS Statistics v. 23.

\section{Prediction testing with online IrisPlex calculator}

All the collected samples were tested with online web-based IrisPlex model (http://hirisplex.erasmusmc.nl/). Probabilities for particular eye colours were generated for the tested 1020 samples considering all six SNPs and position rs12913832 alone (assuming unknown state for the remaining 5 SNPs). Sensitivity and specificity of prediction were calculated separately for males and females, and the dependence of their values and gender was tested using Pearson's $\chi^{2}$ test. Additionally, AUC values for particular eye colours were calculated to compare performance of IrisPlex model and prediction models developed under this study. Due to the fact that IrisPlex tool considers only three eye colour categories, hazel eye colour category was combined in this procedure with brown eye colour category because of their highest similarity [19].

\section{Results}

\section{Study sample characteristics}

The Polish population under study comprised of 535 (52.5\%) individuals of blue eye colour, $127(12.5 \%)$ individuals of green eye colour, 218 (21.4\%) individuals of hazel eye colour and $140(13.7 \%)$ individuals of brown eye colour. The prevalence of blue eye colour was found to be significantly higher in males $(58.1 \%)$ than in females $(48.2 \%)$ with $\chi^{2} p=0.002$. In contrast, females were found more likely to have green eye colour $(14.2 \%)$ comparing to males $(10.0 \%)$ with $\chi^{2}$ $p=0.044$. No significant differences were noted between genders for hazel and brown eye colours $\left(\chi^{2} p=0.532\right.$ and $\chi^{2}$ $p=0.070$, respectively) (Fig. 1).

\section{Eye colour}

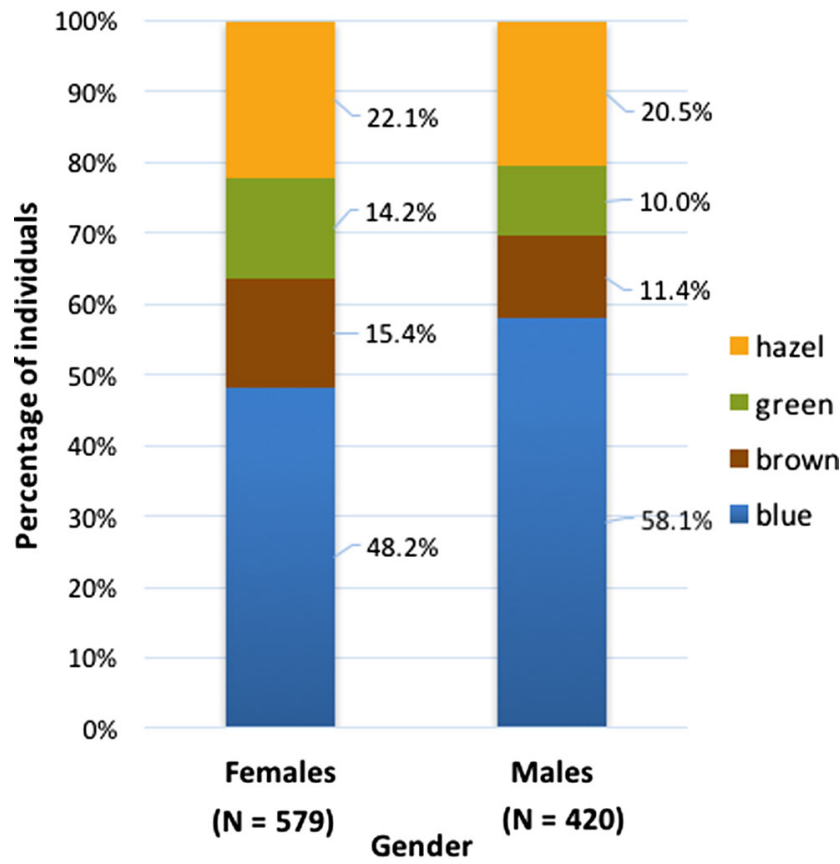

Fig. 1 Eye colour frequencies in females and males in the Polish study sample 


\section{Correlation testing between gender, SNPs and eye colour}

Correlation analysis between particular variables and eye colour showed the strongest effect (correlation coefficient $>0.5$ ) for rs12913832 in HERC2 on blue/green/hazel/brown eye colour as well as blue/non-blue eye colour with $p=5.711 \times 10^{-138}$ and $p=1.189 \times 10^{-108}$, respectively. Small effects (correlation coefficient $0.05-0.2$ ) were noted for rs 1800407 in $O C A 2$, rs12896399 in SLC24A4 and rs16891982 in SLC45A2 with $p=5.508 \times 10^{-4}, p=0.024, p=0.014$, respectively, when four eye colour categories were considered and $p=1.539 \times 10^{-4}$, $p=0.002$ and $p=0.026$, respectively, when blue/non-blue eye colour was studied. No effect of rs1393350 and rs12203592 was noted for both eye colour categorizations. Gender was found to have small size effect on eye colour (correlation coefficient $\sim 0.1$ ) with $p=0.010$ for blue/green/hazel/brown and $p=0.002$ for blue/non-blue eye colour categorization (Table 1).

\section{Power of the sample size}

Theoretical minimal ORs values detectable with a power of at least $80 \%$ in a group of 535 blue and 485 non-blue individuals were calculated to equal $\mathrm{OR}=1.510$ (or 0.627 ) for rs12913832, OR $=1.925$ (or 0.388) for rs 1800407 , $\mathrm{OR}=1.433$ (or 0.692) for $\mathrm{rs} 12896399$, $\mathrm{OR}=2.549$ (or 0.132 ) for $\mathrm{rs} 16891982, \mathrm{OR}=1.509$ (or 0.627 ) for $\mathrm{rs} 1393350$ and $\mathrm{OR}=1.784(0.461)$ for rs12203592 depending on the minor allele frequency.

\section{Association analyses with logistic regression}

Logistic regression was used to test the effect size of association between the tested variables and blue/non-blue eye colour. Firstly, independent effects of seven individual variables were examined in univariate association analyses. Position rs12913832 in HERC2 was confirmed to be the most strongly associated with eye colour with $\mathrm{C}$ allele increasing the odds for blue eye colour by a factor of 32.3 $\left(p=1.961 \times 10^{-72}\right)$. Among six IrisPlex SNPs, significant association was also noted for rs 1800407 in $O C A 2$ $\left(p=4.886 \times 10^{-5}\right)$, rs 12896399 in SLC24A4 $\left(p=5.260 \times 10^{-4}\right)$ and $\mathrm{rs} 16891982$ in SLC45A2 $(p=0.009)$ with $O C A 2$ and $S L C 24 A 4$ polymorphisms significant also after Bonferroni correction. No association was noted for the remaining two polymorphisms, rs 1393350 in $T Y R$ $(p=0.158)$ and rs12203592 in IRF4 $(p=0.675)$. Gender was found to be significantly associated with eye colour with males having $\sim 1.5$ higher odds for blue eye colour comparing to females $(p=0.002)$. Nagelkerke $R^{2}$ for gender was $1.3 \%$ (for blue/non-blue eye colour) which is much lower than established for $\mathrm{rs} 12913832\left(R^{2}=55.6 \%\right)$, lower than calculated for $\operatorname{rs} 1800407\left(R^{2}=2.3 \%\right)$ and $\operatorname{rs} 12896399\left(R^{2}=1.6 \%\right)$ but higher than established for $\operatorname{rs} 16891982\left(R^{2}=0.9 \%\right)$, rs1393350 $\left(R^{2}=0.3 \%\right)$ and $\operatorname{rs} 12203592\left(R^{2}=0.02 \%\right)$. In the next step, all seven variables were tested simultaneously in multivariate association analysis. In this approach, position rs1393350 in TYR was also found to be associated with blue/non-blue eye colour $(p=0.029)$. However, this result was not significant after Bonferroni correction for multiple comparisons. The significance of gender in multivariate association analysis increased to $p=1.480 \times 10^{-4}$ placing gender as the third most significant factor in blue/non-blue eye colour determination after $\operatorname{rs} 12913832\left(p=9.055 \times 10^{-64}\right)$ and rs $12896399\left(p=7.402 \times 10^{-7}\right)$. Variables were additionally ranked using $-2 \log$ likelihood of reduced model statistic confirming gender being the third most important factor among the seven tested in blue/non-blue eye colour

Table 1 Correlation analysis between 6 IrisPlex SNPs, gender and eye colour

\begin{tabular}{|c|c|c|c|c|c|c|c|c|}
\hline \multirow[t]{3}{*}{ Variable } & \multirow[t]{3}{*}{ Gene } & \multirow[t]{3}{*}{$N$} & \multicolumn{6}{|l|}{ Correlation analysis } \\
\hline & & & \multicolumn{3}{|c|}{ Blue/green/hazel/brown eye colour } & \multicolumn{3}{|l|}{ Blue/non-blue eye colour } \\
\hline & & & Correlation coefficient ${ }^{\mathrm{a}}$ & Effect size $^{\mathrm{b}}$ & Pearson $p$ value & Correlation coefficient ${ }^{\mathrm{a}}$ & Effect size $\mathrm{e}^{\mathrm{b}}$ & Pearson $p$ value \\
\hline rs 12913832 & HERC2 & 1020 & $0.566 / 0.766$ & Large & $5.711 \times 10^{-138}$ & $0.698 / 0.810$ & Large & $1.189 \times 10^{-108}$ \\
\hline rs 1800407 & $O C A 2$ & 1020 & $0.108 / 0.185$ & Small & $5.508 \times 10^{-4}$ & $0.131 / 0.184$ & Small & $1.539 \times 10^{-4}$ \\
\hline rs 12896399 & $S L C 24 A 4$ & 1020 & $0.084 / 0.145$ & Small & 0.024 & $0.112 / 0.157$ & Small & 0.002 \\
\hline rs 16891982 & $S L C 45 A 2$ & 1020 & $0.089 / 0.152$ & Small & 0.014 & $0.085 / 0.120$ & Small & 0.026 \\
\hline rs 1393350 & $T Y R$ & 1020 & $0.045 / 0.079$ & None & 0.649 & $0.044 / 0.063$ & None & 0.368 \\
\hline rs 12203592 & IRF4 & 1020 & $0.036 / 0.061$ & None & 0.861 & $0.023 / 0.032$ & None & 0.770 \\
\hline Gender & - & 1017 & $0.105 / 0.148$ & Small & 0.010 & $0.097 / 0.137$ & Small & 0.002 \\
\hline
\end{tabular}

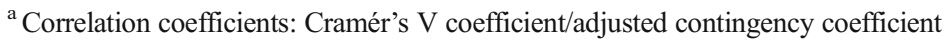

${ }^{\mathrm{b}}$ Effect size: none — $k x$ Cramér's V coefficient $<0.1$, small— $k x$ Cramér's V coefficient $0.1-0.3$, medium — $k x$ Cramér's V coefficient $0.3-0.5$, large — $k$ $x$ Cramér's V coefficient $>0.5$, where $k x$ Cramér's V coefficient is so called Cohen's coefficient and $k$ is equal to the square root of 2 for blue/green/hazel/ brown eye colour and all genes and 1 in other cases [17] 
determination. Gender was ranked as the fourth most important factor (after rs12913832, rs1800407 and rs12896399) when eye colour was categorized as blue/green/hazel/brown (Table 2).

\section{Prediction modeling using neural networks and the complete set of $\mathbf{1 0 2 0}$ individuals}

The entire population of 1020 Polish samples was used to develop neural networks prediction model and evaluate the predictive capacity of IrisPlex SNPs and gender. Variables were sequentially incorporated into the analyses and tested for their impact on accuracy prediction expressed by the value of AUC. As presented in Fig. 2, position rs12913832 in HERC2 alone provided accurate prediction of blue, brown and hazel eye colour at the level of AUC $>0.8$. Noticeable impact on AUC increase ( $>0.01)$ was observed with rs1800407 in OCA2 and rs12896399 in SLC24A4. Position rs1800407 in $O C A 2$ increased the value of AUC for green eye colour (increase by 0.058 for neural networks) and brown eye colour prediction (increase by 0.013 for neural networks). Position rs12896399 in SLC24A4 improved the prediction of blue and green eye colour by increasing the value of AUC by 0.02 and 0.04 , respectively. The remaining three polymorphisms had smaller impact on the accuracy of prediction increasing the value of AUC by less than 0.01 (Fig. 2). The implementation of gender into the neural networks prediction model only marginally impacted the accuracy of the prediction measured by AUC, sensitivity and specificity, and the results were ambiguous. Gender noticeably increased the accuracy of green eye colour prediction with AUC change by 0.04 but with the sensitivity decrease from 0.71 to $0.00 \%$. Similar result was obtained for hazel eye colour (AUC increase by 0.01 and sensitivity decrease by 1.28 percentage points (pp)). Slight decrease in AUC (by 0.026), sensitivity (by $1.55 \mathrm{pp}$ ) and specificity (by $0.49 \mathrm{pp}$ ) values was noted for blue eye colour when gender was considered. In turn, increase in AUC (by 0.001) and prediction sensitivity (by $0.81 \mathrm{pp}$ ) values was noted for brown eye colour category (Fig. 2, Table 3). The small impact of gender on eye colour prediction was also noted when multinomial logistic regression was used with the increase in AUC value for all eye colour categories (change from 0.872 to 0.880 for blue, 0.611 to 0.628 for intermediate, 0.797 to 0.800 for hazel and 0.889 to 0.892 for brown eye colour) but without changes in sensitivity and specificity prediction parameters values (Table 3). Importantly, neural networks method in general provided higher values of AUC comparing to logistic regression with AUC increase from 0.872 to 0.889 for blue eye colour, from 0.611 to 0.667 for green eye colour, from 0.797 to 0.833 for hazel eye colour and from 0.889 to 0.917 for brown eye colour category (Fig. 2, Table 3).

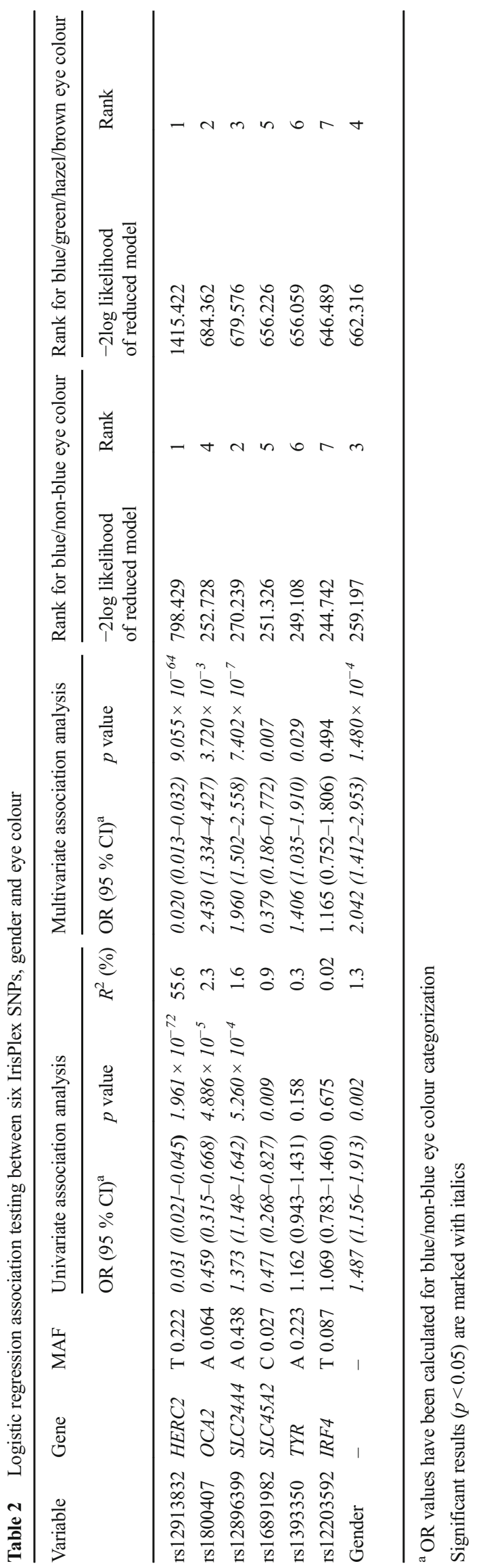


Fig. 2 Contribution of six IrisPlex SNPs and gender into the overall accuracy of eye colour prediction measured by AUC. Values of AUC have been calculated for neural networks

(a), multinomial logistic regression (b) and IrisPlex model (c)

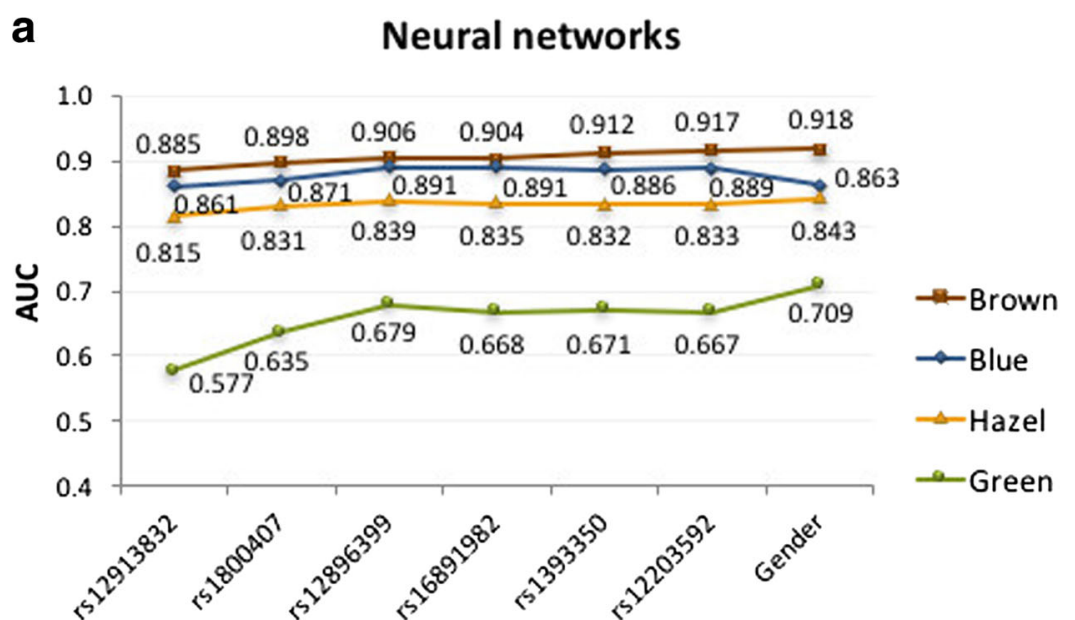

Variables sequentially implemented in prediction model

b

\section{Logistic regression}

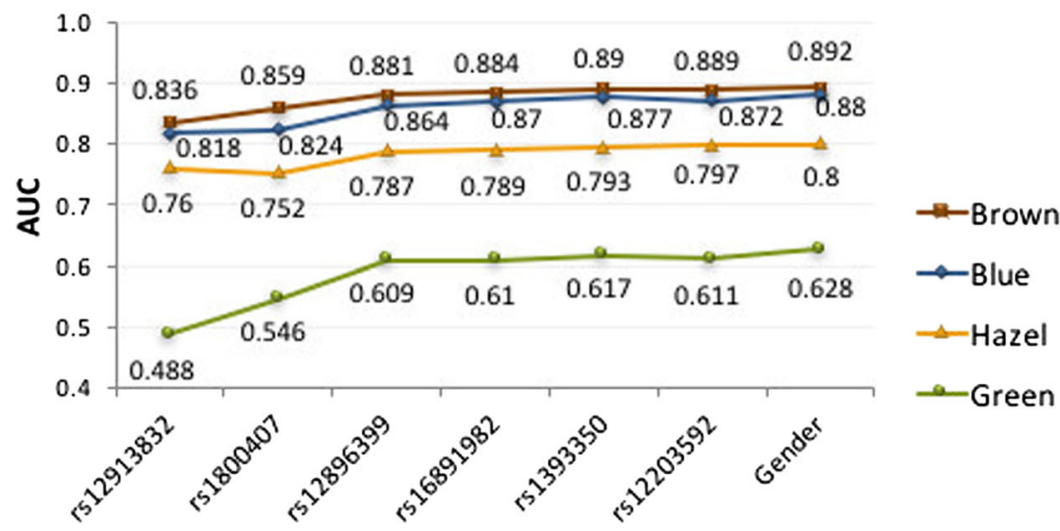

Variables sequentially implemented in prediction model

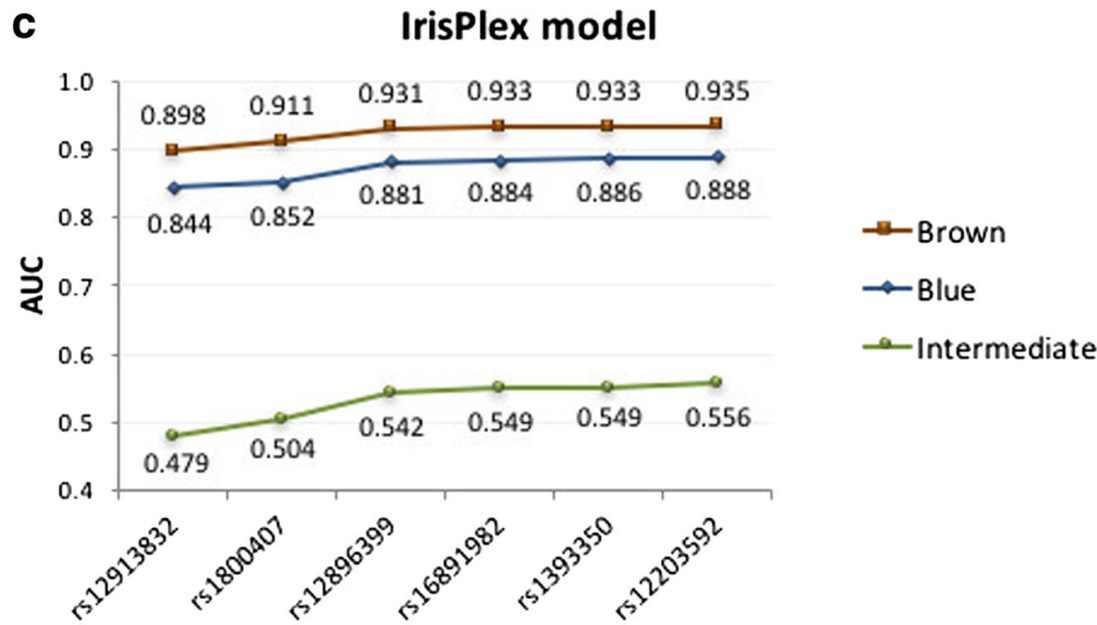

Variables sequentially implemented in prediction model 
Table 3 Impact of gender on the performance of eye colour prediction models developed with a Polish sample set using neural networks and multinomial logistic regression methods

\begin{tabular}{|c|c|c|c|c|c|}
\hline \multirow[t]{3}{*}{ Eye colour category } & \multirow[t]{3}{*}{ Prediction accuracy } & \multicolumn{4}{|l|}{ Mathematical method } \\
\hline & & \multicolumn{2}{|l|}{ Neural networks } & \multicolumn{2}{|c|}{ Multinomial logistic regression } \\
\hline & & Gender not included & Gender included & Gender not included & Gender included \\
\hline \multirow[t]{3}{*}{ Blue eye colour } & AUC & 0.889 & 0.863 & 0.872 & 0.880 \\
\hline & Sensitivity [\%] & 94.06 & 92.51 & 93.11 & 93.11 \\
\hline & Specificity $[\%]$ & 74.34 & 73.85 & 74.07 & 74.07 \\
\hline \multirow[t]{3}{*}{ Green eye colour } & AUC & 0.667 & 0.709 & 0.611 & 0.628 \\
\hline & Sensitivity [\%] & 0.71 & 0.00 & 0.00 & 0.00 \\
\hline & Specificity [\%] & 99.55 & 99.88 & 99.88 & 99.88 \\
\hline \multirow[t]{3}{*}{ Hazel eye colour } & AUC & 0.833 & 0.843 & 0.797 & 0.800 \\
\hline & Sensitivity $[\%]$ & 64.88 & 63.60 & 65.20 & 65.20 \\
\hline & Specificity $[\%]$ & 81.04 & 79.91 & 80.22 & 80.22 \\
\hline \multirow[t]{3}{*}{ Brown eye colour } & AUC & 0.917 & 0.918 & 0.889 & 0.892 \\
\hline & Sensitivity [\%] & 34.23 & 35.04 & 33.80 & 33.80 \\
\hline & Specificity $[\%]$ & 95.54 & 94.18 & 94.52 & 94.52 \\
\hline
\end{tabular}

\section{Prediction testing using IrisPlex online tool}

Gender has been suggested to explain discrepancies in eye colour prediction based on rs12913832 position in HERC2 [9]. Therefore, we analysed the distribution of genotypes in males and females and found that among males of CC genotype $85.7 \%$ have blue eye colour but significantly lower proportion of blue eye colour $(77 \%)$ has been found in females of CC genotype ( $p=0.007)$ (Fig. 3). To further evaluate the effect of gender on eye colour prediction, we tested all 1020 samples with available online IrisPlex model. Higher level of prediction sensitivity of blue eye colour was observed in females $(95.1 \%)$ comparing to males $(91.8 \%)$ when using genotype data for all six SNPs, and similar result was obtained when the prediction was performed based on rs12913832 position alone (94.1\% of sensitivity prediction in females, $91.0 \%$ of sensitivity prediction in males). However, in both calculations, the results were insignificant $(p=0.117$ and $p=0.170$, respectively). Moreover, higher level of specificity of blue eye colour prediction was shown in males (76.1 or $79 \%$ when using six IrisPlex SNPs or rs12913832) comparing to females (71.8 or $73.8 \%$ when using six IrisPlex SNPs or rs12913832), but the results were also insignificant. Overall, no significant differences in eye colour prediction success were observed between genders across all eye colour categories (Tables 4 and 5). IrisPlex model provided similar accuracy of prediction for blue eye colour when comparing to logistic regression and neural networks models developed in this study (IrisPlex $\mathrm{AUC}=0.888$, neural networks $=0.889$, logistic regression $=0.872$ ), slightly higher value of AUC for brown eye colour (IrisPlex AUC $=0.935$, neural networks $\mathrm{AUC}=0.917$, logistic regression $\mathrm{AUC}=0.889$ ) but lower

\section{Eye colour}
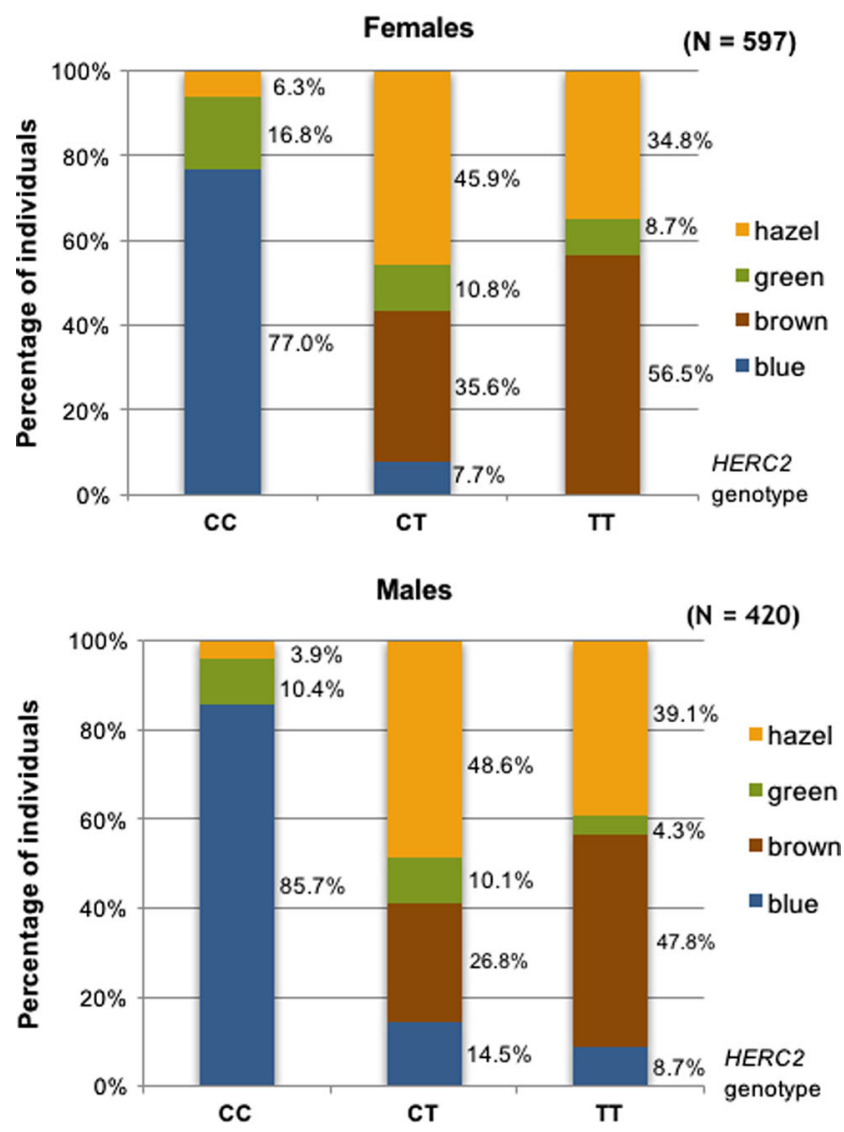

Fig. 3 Distribution of eye colour categories in individuals of CC, CT and TT genotype in rs 12913832 HERC2 compared in males and females 
Table 4 Success rate of eye colour prediction in females and males based on six IrisPlex SNPs (IrisPlex online tool)

Prediction success based on IrisPlex model

\begin{tabular}{|c|c|c|c|c|c|c|}
\hline \multirow[t]{2}{*}{ Eye colour category } & \multicolumn{3}{|l|}{ Sensitivity \% } & \multicolumn{3}{|l|}{ Specificity \% } \\
\hline & Females & Males & $\chi^{2} p$ value & Females & Males & $\chi^{2} p$ value \\
\hline Blue & $95.1(274 / 288)$ & $91.8(224 / 244)$ & 0.117 & $71.8(222 / 309)$ & $76.1(134 / 176)$ & 0.304 \\
\hline Intermediate & $0.0(0 / 85)$ & $0.0(0 / 42)$ & - & $100.0(512 / 512)$ & $100.0(378 / 378)$ & - \\
\hline Brown & $88.0(197 / 224)$ & $91.0(122 / 134)$ & 0.363 & $89.5(334 / 373)$ & $88.8(254 / 286)$ & 0.797 \\
\hline
\end{tabular}

value for green (intermediate) eye colour prediction (IrisPlex $\mathrm{AUC}=0.556$, neural networks $\mathrm{AUC}=0.667$, logistic regression $\mathrm{AUC}=0.611$ ) (Fig. 2).

\section{Discussion}

DNA-based prediction of human appearance is a very promising approach that can be useful for investigations of biological traces and human remains when other DNA analysis methods fail to identify a suspect. However, high complexity of genetic basis underlying human appearance traits makes this field very difficult. The genetic prediction of human eye colour is the most thoroughly studied and the most advanced. The first eye colour prediction attempts have been reported before genome-wide association studies (GWAS) identifying large number of pigmentation-associated loci [20, 21]. The discovery of rs 12913832 in HERC2 has been crucial [22-24]. This SNP is believed to be a key eye colour regulator influencing $O C A 2$ expression. So far, the IrisPlex system is the most validated tool for eye colour prediction $[4,5,12,13$, 19, 25-28]. This method is based on six eye colour prediction SNPs $[6,18]$ and provides high level of prediction accuracy for blue and brown eye colour at $\sim 90 \%$ with much lower accuracy level obtained for intermediate irises.

The IrisPlex validation studies, which have involved various populations, provided unprecedented insight into the genetics of the six IrisPlex predictors revealing significant interpopulation differences in prediction capacity of particular SNPs. The key role of rs12913832 in eye colour prediction is undisputable, and clearly this marker is included in all available eye colour prediction models [6, 8, 10, 29]. The role of rs1800407 in OCA2 has also been emphasized by many research reports [20, 30-35]. The effect of rs12896399 on eye colour determination discovered in a GWA study from 2007 [36] has also been confirmed in several studies performed on northern and southern European populations [8-11, 18]. Significance of rs16891982 in SLC45A2 seems to depend on a minor allele frequency in a study population as it is lower in northern Europe populations (MAF 0.04, 1000 genomes) and higher in southern Europe (MAF 0.18, 1000 genomes). Despite its low frequency in Poland $(\mathrm{MAF}=0.027)$, its effect has been detected in the present study at the level of $\mathrm{OR}=0.471$ (or 2.12) for blue/non-blue eye colour but with lower significance than observed for rs12913832, rs1800407 and rs12896399. The role of the remaining two positions, namely rs1393350 and rs12203592 is the most puzzling as the MAF in each case is rather high $(\mathrm{MAF}=0.24$ for rs1393350 and 0.12 for rs12203592, 1000 genomes) while the pattern of association is ambiguous. These two SNPs were not associated with eye colour in our previous study involving smaller population sample [11]. In the present study, position rs1393350 was associated with blue/non-blue eye colour only in a multivariate association analysis, and the result was insignificant after correction for multiple comparisons. A weak association of TYR rs1393350 and the lack of association in case of IRF4 rs12203592 with eye colour have been reported in other study exploring several European populations [8]. IRF4 has not been included in an eye colour classification tree developed by Allwood et al. based on a population sample

Table 5 Success rate of eye colour prediction in females and males based on rs12913832 (IrisPlex online tool)

Prediction success based on rs 12913832

\begin{tabular}{|c|c|c|c|c|c|c|}
\hline \multirow[t]{2}{*}{ Eye colour category } & \multicolumn{3}{|l|}{ Sensitivity \% } & \multicolumn{3}{|l|}{ Specificity \% } \\
\hline & Females & Males & $\chi^{2} p$ value & Females & Males & $\chi^{2} p$ value \\
\hline Blue & $94.1(271 / 288)$ & $91.0(222 / 244)$ & 0.170 & $73.8(228 / 309)$ & $79.0(139 / 176)$ & 0.200 \\
\hline Intermediate & $0.0(0 / 85)$ & $0.0(0 / 42)$ & - & $100.0(512 / 512)$ & $100.0(378 / 378)$ & - \\
\hline Brown & $90.2(202 / 224)$ & $92.5(124 / 134)$ & 0.449 & $88.5(330 / 373)$ & $87.1(249 / 286)$ & 0.583 \\
\hline
\end{tabular}


from New Zealand while the model involves rs1393350 in TYR [10]. Lack of association for these two SNPs has also been revealed in a recent study performed on a Portuguese population [14]. The examination of rs 12203592 in IRF4 in 12 European and Asian populations has revealed very weak effect of this SNP for iris colour prediction [12]. The minor allele frequency for rs 12203592 in our population is lower $(\mathrm{MAF}=0.087)$ than assessed for global European population (MAF $=0.12,1000$ genomes) which may be the reason for the lack of association detected. However, association at the level of OR 2.0 was discovered for rs1800407 and rs16891982 characterized by even lower values of MAF ( 0.064 and 0.027 , respectively). Study performed on a Spanish population sample has revealed marginally significant association of rs12203592 in IRF4 with eye colour with the effect size of OR $\sim 2.12$ when adjusted for the remaining IrisPlex SNPs [9]. Our study sample was theoretically sufficient to detect association of IRF4 assuming OR $\geq 1.78$. Thus, OR $\sim 1.17$ may suggest the problem with statistical power to detect association between eye colour and rs12203592 in this study. Interestingly, IRF4 shows ambiguous pattern of association with cutaneous cancers in various populations [37-41]. Pietroni et al. has reported three IrisPlex SNPs to be the only informative for eye colour prediction, and this set comprised rs12913832 in HERC2, rs1800407 in OCA2 and rs16891982 in SLC45A2 [13]. In our study, position rs12896399 in SLC24A4 excluded in that study was ranked as the second most important factor for blue/non-blue eye colour and the third most important factor for blue/green/hazel/brown eye colour determination. The increase in AUC value illustrating accuracy of prediction was the most noticeable after the implementation of rs12913832 in HERC2, rs1800407 in OCA2 and rs12896399 in SLC24A4 into the developed prediction models and was significantly lower when the remaining three IrisPlex predictors were added. These results strongly support existence of inter-population differences in association patterns in Europe and that different polymorphisms may have different predictive power in different populations. Therefore, we conclude that the set of six SNP predictors selected in IrisPlex model is a good minimal set for eye colour prediction when dealing with a sample of unknown biogeographic ancestry. These inter-population differences are even more severe at the worldwide scale. Blue eye colour is mainly limited to European populations, but there is a strong evidence for convergent evolution of skin pigmentation in Europeans and Asians and significant role of different DNA variants within $H E R C 2-O C A 2$ region responsible for skin lightening in Europe and Asia [42-44]. Interestingly, inter-population differences in the prevalence of various $O C A 2$ alleles were recently shown in the region of East Asia, and the data suggested that the studied polymorphisms might have been selected independently in various populations of East Asia [45]. This observation further emphasizes possible population-specific role of various pigmentation related DNA variants.

Moreover, the studies have also disclosed a role of gender for eye colour prediction accuracy which can be population specific $[9,13]$. Although, gender has been found not to improve prediction of eye colour when incorporated into the original IrisPlex model [15], and further studies of this subject involving various populations can be intriguing. There are few studies suggesting that males tend to have lighter eye colour comparing to females. The prevalence of blue eye colour in a Spanish population is quite low, and recently significantly lower proportion of blue-eyed females $(8.5 \%)$ has been reported comparing to blue-eyed males (14.7\%). In turn, Spanish brown-eyed females have been found to be more common $(78.5 \%)$ comparing to Spanish brown-eyed males $(71.4 \%)$ [9]. Gender effect on quantitative eye colour variation (hue and saturation) has also been noted in the study performed on the Dutch Europeans [46]. The association of gender with quantitative eye colour variation has also been reported for the Italian study sample also suggesting that females tend to have darker eye colour than males. However, in the same study, no association of gender with eye colour variation has been observed in Danish and Swedish population samples [13]. Our data indicated significantly higher proportion of blue-eyed Polish males (58.1\%) comparing to blueeyed Polish females (48.2\%). Consequently, Polish females were found more likely to have green eye colour (14.2\%) comparing to males $(10.0 \%)$, but there were no significant differences between genders for hazel and brown eye colours. In our previous study, higher proportion of Polish blue-eyed males $(60.9 \%)$ has been reported comparing to blue-eyed females $(52.3 \%)$, but the result has been insignificant which may be explained by significantly lower number of samples analysed $(\mathrm{N}=388)$ [47]. In this large dataset, gender was found to be significantly associated with eye colour, but small size effect was reported with males having $\sim 1.5$ higher odds for blue eye colour comparing to females. Gender was revealed to explain $1.3 \%$ of variation in blue/non-blue eye colour which is higher than reported in the Dutch population $(0.1 \%)$ [15] but lower than calculated for the Italian sample (4.9\%) [13]; however, in both cases, calculations have been performed for quantitative eye colour. In the multivariate logistic regression analysis covering six IrisPlex SNPs and gender, gender was ranked as third (when blue/non-blue eye colour has been considered) or fourth (when blue/green/hazel/ brown eye colour has been considered) most important factor.

Identified association of gender with eye colour variation has raised a dispute on its possible impact on eye colour prediction performance. The authors of the study performed on the Spanish population have noted that among CC homozygotes for HERC2 rs12913832, which is believed to be a strong predictor of blue eye colour, $79 \%$ of males were indeed blueeyed and only $54 \%$ of females were blue-eyed suggesting that 
females tend to have darker eye colour than males when dealing with CC genotype in HERC2 [9]. Similar result but with lower disproportion was obtained in the present study. Among Polish rs12913832 CC homozygotes, $85.7 \%$ of males were found to have blue eye colour, and significantly lower proportion of blue eye colour was observed in females with $77 \%$. Contrary to the results obtained for the Spanish sample [9], the authors of IrisPlex model have not observed the differences in eye colour prediction success between males and females when analyzed much larger number of samples including $>5300$ Dutch Europeans and >3800 Europeans. They also have not observed the improvement of eye colour prediction when gender has been incorporated into the original IrisPlex model [15]. In the present study, higher sensitivity level of blue eye colour prediction was obtained in females comparing to males, and the opposite effect was observed in respect to the specificity level but the results were insignificant. Moreover, the incorporation of gender into the neural networks model developed in our study had ambiguous impact on the prediction efficiency with small AUC increase detected for green, hazel and brown eye colour but decrease observed for blue eye colour. These results confirm that the effect of gender on eye colour prediction is rather small in the studied population.

It is unclear how gender can affect the differences in human eye colour variation, and no genes on human gender chromosomes are known to be associated with pigmentation. Moreover, the effect of gender on eye colour variation seems to be complex as it appears to be population specific, with stronger effect noted in southern Europe populations. It is also worth noting that most of the reported European genome-wide association studies on pigmentation have been conducted on northern European populations [22, 36, 42, 48, 49]. Therefore, it seems that more extensive studies on the genetics of pigmentation exploring southern European populations are needed in order to clarify the contribution of gender into the eye colour variation in humans. Additional research is also needed to verify the impact of gender on eye colour prediction in various populations including admixed population samples.

Besides, verification of the list of predictors included into the eye colour prediction models also different mathematical approaches could be tested as they may give different results. So far, multinomial logistic regression [6, 18], Bayesian approach $[8,19]$ and classification trees $[10,29]$ have been used to develop eye-colour prediction models. In this study, neural networks have been explored and provided higher values of AUC comparing to logistic regression corroborating our recent observation reported for the prediction of hair morphology in humans that neural networks approach may be a good alternative for the traditional parametric methods [50]. Comparison of our neural networks model with the multinomial logistic regression IrisPlex model indicated slightly lower prediction accuracy of brown eye colour but noticeably higher accuracy of green eye colour prediction using neural networks approach. Since the discovery sample set used to build NN model was smaller $(\sim 1000)$ than for the IrisPlex model ( $\sim 10000)$, further studies are necessary to explore this problem more thoroughly.

In conclusion, HERC2, OCA2, SLC24A4 and SLC45A2 were found to be significantly associated with eye colour in the studied Polish population. Gender was ranked as the third most important factor in blue/non-blue eye colour determination. Its effect size was found to be small with males having $\sim 1.5$ higher odds for blue eye colour comparing to females, and the observed impact on eye colour prediction was small. The obtained results provided further evidence that the genetics of eye colour is population specific and indicates that further studies on eye colour prediction involving various population samples, and more complex mathematical approaches will be intriguing.

Acknowledgments The work leading to these results was financially supported from the European Union Seventh Framework Programme (FP7/2007-2013) under grant agreement no. 285487 (EUROFORGEN$\mathrm{NoE})$.

Compliance with ethical standards Written informed consent was obtained from all the samples donors, and the study protocol was approved by the Ethics Committee of the Jagiellonian University in Krakow (KBET/17/B/2005) and the Commission on Bioethics of the Regional Board of Medical Doctors in Krakow (48 KBL/OIL/2008).

Open Access This article is distributed under the terms of the Creative Commons Attribution 4.0 International License (http:// creativecommons.org/licenses/by/4.0/), which permits unrestricted use, distribution, and reproduction in any medium, provided you give appropriate credit to the original author(s) and the source, provide a link to the Creative Commons license, and indicate if changes were made.

\section{References}

1. Kayser M, Schneider PM (2009) DNA-based prediction of human externally visible characteristics in forensics: motivations, scientific challenges, and ethical considerations. Forensic Sci Int Genet 3: 154-161. doi:10.1016/j.fsigen.2009.01.012

2. Branicki W, Pośpiech E, Kupiec T, Styrna J (2014) A new dimension of the forensic DNA expertise - the need for training experts and expertise recipients. Arch Med Sadowej Kryminol 64:175-194

3. Kayser M (2015) Forensic DNA phenotyping: predicting human appearance from crime scene material for investigative purposes. Forensic Sci Int Genet 18:33-48. doi:10.1016/j.fsigen.2015.02.003

4. Walsh S, Chaitanya L, Clarisse L et al (2014) Developmental validation of the HIrisPlex system: DNA-based eye and hair colour prediction for forensic and anthropological usage. Forensic Sci Int Genet 9:150-161. doi:10.1016/j.fsigen.2013.12.006

5. Chaitanya L, Walsh S, Andersen JD et al (2014) Collaborative EDNAP exercise on the IrisPlex system for DNA-based prediction of human eye colour. Forensic Sci Int Genet 11:241-251. doi:10. 1016/j.fsigen.2014.04.006

6. Walsh S, Liu F, Ballantyne KN et al (2011) IrisPlex: a sensitive DNA tool for accurate prediction of blue and brown eye colour in 
the absence of ancestry information. Forensic Sci Int Genet 5:170 180. doi:10.1016/j.fsigen.2010.02.004

7. Visser M, Kayser M, Palstra RJ (2012) HERC2 rs12913832 modulates human pigmentation by attenuating chromatin-loop formation between a long-range enhancer and the OCA2 promoter. Genome Res 22:446-455. doi:10.1101/gr.128652.111

8. Ruiz Y, Phillips C, Gomez-Tato A et al (2013) Further development of forensic eye color predictive tests. Forensic Sci Int Genet 7:2840. doi:10.1016/j.fsigen.2012.05.009

9. Martinez-Cadenas C, Peña-Chilet M, Ibarrola-Villava M, Ribas G (2013) Gender is a major factor explaining discrepancies in eye colour prediction based on HERC2/OCA2 genotype and the IrisPlex model. Forensic Sci Int Genet 7:453-460. doi:10.1016/j. fsigen.2013.03.007

10. Allwood JS, Harbison S (2013) SNP model development for the prediction of eye colour in New Zealand. Forensic Sci Int Genet 7: 444 452. doi:10.1016/j.fsigen.2013.03.005

11. Pośpiech E, Wojas-Pelc A, Walsh S et al (2014) The common occurrence of epistasis in the determination of human pigmentation and its impact on DNA-based pigmentation phenotype prediction. Forensic Sci Int Genet 11:64-72. doi:10.1016/j.fsigen.2014.01.012

12. Yun L, Gu Y, Rajeevan H, Kidd KK (2014) Application of six IrisPlex SNPs and comparison of two eye color prediction systems in diverse Eurasia populations. Int J Legal Med 128:447-453. doi: 10.1007/s00414-013-0953-1

13. Pietroni C, Andersen JD, Johansen P et al (2014) The effect of gender on eye colour variation in European populations and an evaluation of the IrisPlex prediction model. Forensic Sci Int Genet 11:1-6. doi:10.1016/j.fsigen.2014.02.002

14. Dario P, Mouriño H, Oliveira AR et al (2015) Assessment of IrisPlex-based multiplex for eye and skin color prediction with application to a Portuguese population. Int J Legal Med 129: 1191-1200. doi:10.1007/s00414-015-1248-5

15. Liu F, Walsh S, Kayser M (2014) Of gender and IrisPlex eye colour prediction: a reply to Martinez-Cadenas et al. Forensic Sci Int Genet 9:e5-e6. doi:10.1016/j.fsigen.2013.06.006

16. Pośpiech E, Draus-Barini J, Kupiec T et al (2011) Gene-gene interactions contribute to eye colour variation in humans. J Hum Genet 56:447-55. doi:10.1038/jhg.2011.38

17. Sheskin DJ (2011) Handbook of parametric and nonparametric statistical procedures. Chapman\&Hall/CRC, Boca Raton, pp 675-676

18. Liu F, van Duijn K, Vingerling JR et al (2009) Eye color and the prediction of complex phenotypes from genotypes. Curr Biol 19: R192-R123. doi:10.1016/j.cub.2009.01.027

19. Pośpiech E, Draus-Barini J, Kupiec T et al (2012) Prediction of eye color from genetic data using Bayesian approach. J Forensic Sci 57: 880-886. doi:10.1111/j.1556-4029.2012.02077.x

20. Frudakis T, Thomas M, Gaskin Z et al (2003) Sequences associated with human iris pigmentation. Genetics 165:2071-283

21. Frudakis T, Terravainen T, Thomas M (2007) Multilocus OCA2 genotypes specify human iris colors. Hum Genet 122:311-326. doi:10.1007/s00439-007-0401-8

22. Kayser M, Liu F, Janssens ACJW et al (2008) Three genome-wide association studies and a linkage analysis identify HERC2 as a human iris color gene. Am J Hum Genet 82:411-423. doi:10. 1016/j.ajhg.2007.10.003

23. Sturm RA, Duffy DL, Zhao ZZ et al (2008) A single SNP in an evolutionary conserved region within intron 86 of the HERC2 gene determines human blue-brown eye color. Am J Hum Genet 82:424 431. doi:10.1016/j.ajhg.2007.11.005

24. Eiberg H, Troelsen J, Nielsen M et al (2008) Blue eye color in humans may be caused by a perfectly associated founder mutation in a regulatory element located within the HERC2 gene inhibiting OCA2 expression. Hum Genet 123:177-187. doi:10.1007/s00439007-0460-x
25. Walsh S, Lindenbergh A, Zuniga SB et al (2011) Developmental validation of the IrisPlex system: determination of blue and brown iris colour for forensic intelligence. Forensic Sci Int Genet 5:464-471. doi:10.1016/j.fsigen.2010. 09.008

26. Kastelic V, Pośpiech E, Draus-Barini J et al (2013) Prediction of eye color in the Slovenian population using the IrisPlex SNPs. Croat Med J 54:381-6

27. Dembinski GM, Picard CJ (2014) Evaluation of the IrisPlex DNA-based eye color prediction assay in a United States population. Forensic Sci Int Genet 9:111-117. doi:10.1016/ j.fsigen.2013.12.003

28. Andersen JD, Johansen P, Harder S et al (2013) Genetic analyses of the human eye colours using a novel objective method for eye colour classification. Forensic Sci Int Genet 7:508-515. doi:10. 1016/j.fsigen.2013.05.003

29. Spichenok O, Budimlija ZM, Mitchell AA et al (2011) Prediction of eye and skin color in diverse populations using seven SNPs. Forensic Sci Int Genet 5:472-478. doi:10.1016/ j.fsigen.2010.10.005

30. Eiberg H, Mohr J (1996) Assignment of genes coding for brown eye colour (BEY2) and brown hair colour (HCL3) on chromosome 15q. Eur J Hum Genet 4:237-241

31. Sturm RA, Teasdale RD, Box NF (2001) Human pigmentation genes: identification, structure and consequences of polymorphic variation. Gene 277:49-62

32. Rebbeck TR, Kanetsky PA, Walker AH et al (2002) P gene as an inherited biomarker of human eye color. Cancer Epidemiol Biomarkers Prev 11:782-784

33. Zhu G, Evans DM, Duffy DL et al (2004) A genome scan for eye color in 502 twin families: most variation is due to a QTL on chromosome 15q. Twin Res 7:197-210. doi:10. $1375 / 136905204323016186$

34. Duffy DL, Montgomery GW, Chen W et al (2007) A threesingle-nucleotide polymorphism haplotype in intron 1 of OCA2 explains most human eye-color variation. Am J Hum Genet 80:241-252. doi:10.1086/510885

35. Branicki W, Brudnik U, Kupiec T et al (2008) Association of polymorphic sites in the OCA2 gene with eye colour using the tree scanning method. Ann Hum Genet 72:184-192. doi:10.1111/j. 1469-1809.2007.00407.x

36. Sulem P, Gudbjartsson DF, Stacey SN et al (2007) Genetic determinants of hair, eye and skin pigmentation in Europeans. Nat Genet 39:1443-152. doi:10.1038/ng.2007.13

37. Duffy DL, Zhao ZZ, Sturm RA et al (2010) Multiple pigmentation gene polymorphisms account for a substantial proportion of risk of cutaneous malignant melanoma. J Invest Dermatol 130:520-528. doi:10.1038/jid.2009.258

38. Duffy DL, Iles MM, Glass D et al (2010) IRF4 variants have agespecific effects on nevus count and predispose to melanoma. Am J Hum Genet 87:6-16. doi:10.1016/j.ajhg.2010.05.017

39. Peña-Chilet $M$, Blanquer-Maceiras $M$, Ibarrola-Villava $M$ et al (2013) Genetic variants in PARP1 (rs3219090) and IRF4 (rs12203592) genes associated with melanoma susceptibility in a Spanish population. BMC Cancer 13:160. doi: 10.1186/1471-2407-13-160

40. Kosiniak-Kamysz A, Pośpiech E, Wojas-Pelc A et al (2012) Potential association of single nucleotide polymorphisms in pigmentation genes with the development of basal cell carcinoma. J Dermatol 39:693-698. doi:10.1111/j.1346-8138. 2012.01559.x

41. Kosiniak-Kamysz A, Marczakiewicz-Lustig A, Marcińska M et al (2014) Increased risk of developing cutaneous malignant melanoma is associated with variation in pigmentation genes and VDR, and may involve epistatic effects. Melanoma Res 24:388-396. doi:10. 1097/CMR.0000000000000095 
42. Norton HL, Kittles RA, Parra E et al (2007) Genetic evidence for the convergent evolution of light skin in Europeans and East Asians. Mol Biol Evol 24:710-722. doi:10.1093/molbev/msl203

43. Donnelly MP, Paschou P, Grigorenko E et al (2012) A global view of the OCA2-HERC2 region and pigmentation. Hum Genet 131:683-696. doi:10.1007/s00439-011-1110-x

44. Yang Z, Zhong H, Chen J et al (2016) A genetic mechanism for convergent skin lightening during recent human evolution. Mol Biol Evol 33:1177-1787. doi:10.1093/molbev/msw003

45. Murray N, Norton HL, Parra EJ (2015) Distribution of two OCA2 polymorphisms associated with pigmentation in EastAsian populations. Hum Genome Var 2(15058):1-5. doi:10. 1038/hgv.2015.58

46. Liu F, Wollstein A, Hysi PG et al (2010) Digital quantification of human eye color highlights genetic association of three new loci. PLoS Genet 6:1-15. doi:10.1371/journal.pgen.1000934
47. Branicki W, Brudnik U, Wojas-Pelc A (2009) Interactions between HERC2, OCA2 and MC1R may influence human pigmentation phenotype. Ann Hum Genet 73:160-170. doi:10.1111/j.14691809.2009.00504.x

48. Sulem P, Gudbjartsson DF, Stacey SN et al (2008) Two newly identified genetic determinants of pigmentation in Europeans. Nat Genet 40:835-7. doi:10.1038/ng.160

49. Liu F, Visser M, Duffy DL et al (2015) Genetics of skin color variation in Europeans: genome-wide association studies with functional follow-up. Hum Genet 134:823-835. doi:10.1007/s00439015-1559-0

50. Pośpiech E, Karłowska-Pik J, Marcińska M et al (2015) Evaluation of the predictive capacity of DNA variants associated with straight hair in Europeans. Forensic Sci Int Genet 19:280-288. doi:10.1016/ j.fsigen.2015.09.004 